\title{
Explaining Ginzburg Landau Equations from First Principles
}

\section{Koutandos $\mathrm{S}^{*}$}

Ekfe Chiou, Agia Paraskevi, Greece

*Corresponding author: Spiros Koutandos, EKFE CHIOU, Maniakiou 17, Agia Paraskevi, Attica 15343, Greece, Tel: 6980129686; Email: skoutandos1@gmail.com

\section{Research Article}

Volume 5 Issue 1

Received Date: January 23, 2021

Published Date: February 02, 2021

\section{Abstract}

We attempt to prove Ginzburg Landau equations given in the field of superconductivity as a result of inspiration. Our view is that they can be derived from thermodynamic arguments through assigning hidden variables to the formalism of quantum mechanics. A coefficient of efficiency is used to derive results and the formula for pressure in quantum mechanics in general is found. The volume is changing due to a spacetime curvature.

Keywords: Quantum thermodynamics; Superconductivity; Free energy; Quantum mechanics

\section{Introduction}

In exposing the physical meaning of Ginzburg-Landau equations we will first need some results of previous work of the author Koutandos S [1,2] which we shall put forth. We are also going to need some formalism taken from standard thermodynamics.

The Gibbs potential is written as:

$$
\Omega=-P V
$$

The rest of the thermodynamic quantities are:

$$
H=U+P V \Rightarrow d H=T d S-P d V+P d V+V d P=T d S+V d P
$$

$$
\begin{gathered}
F=U-T S \Rightarrow d F=P d V-S d T \text { (2) } \\
G=U-T S+P V \Rightarrow G=F-\Omega \Rightarrow d G=V d P-S d T
\end{gathered}
$$

The bulk modulus or the inverse of compressibility of a material is given by the following formula:

$$
B=V \frac{d P}{d V}
$$

The letter $\mathrm{P}$ in quantum mechanics comes from Probability as we know and may be the key for unlocking the formalism. In the article of reference Koutandos S [1] we had found that electrons may be considered as swirling droplets where there is a difference of pressures between their interior and exterior. All we have to do is apply Laplace formula:

$$
\frac{\hbar^{2}}{2 m} \Delta P=\frac{\hbar^{2}}{2 m N} \nabla^{2}|\psi|^{2}=E P-P U-\frac{1}{2 N} \vec{p}^{*} \cdot \vec{v}=B=\sigma K
$$

In equation (6) sigma is a point surface tension of these bubbles and $\mathrm{K}$ is a curvature of spacetime [1,2]. The work of Beig and Simon [3] would apply in this case if we consider that the mass potential is the created volume $\mathrm{V}$ in our case:

$$
\int V d P=c^{2} \int \frac{d m}{\chi}(7)
$$

In equation (7) chi stands for the dielectric susceptibility which alters the speed of light.

Our findings $[1,2]$ suggest that the curvature which appears in the metric of spacetime should be:

$$
\left.K \chi(\vec{r})=\frac{d S}{d V} 98\right)
$$




\section{Main Part}

In superconducting materials as is well known, electron superconductivity is mediated by the interaction of the electrons with the atoms of the structure with the intervention of phonons which are the waves of volume expansion and dilation. The current shows a zero resistance and magnetic flux is partially or totally expelled below a temperature threshold $\mathrm{T}_{c^{\prime}}$. As we have shown [1], the potential energy is purely magnetic.

In the paper we published this theory [2] we expand part of the free energy density of the superconducting electrons as given in superconductor standard books by Tinkham $\mathrm{M}$ [4]:

$$
\frac{d G}{d V} \approx B=\alpha \frac{|\psi|^{2}}{T_{C}}\left(T-T_{C}\right)-h_{c}{ }^{2}-\frac{\hbar^{2}}{2 m V}|(\nabla-i A) \psi|^{2} \text { (9) }
$$

Equation (9) is just an implication of formula (6) if we recall that in quantum mechanics the momentum is given by:

$$
\vec{p}=(-i \hbar \nabla-\vec{A}) \psi
$$

In the next article we shall refer the reader to Koutandos $S$ [5] we had discovered that in critical phenomena such as is the formation of a thin shell between the liquid and its evaporating vapor during boiling we have a Carnot-like description of a reversible process. We had speculated then that tiny droplets may be formed which would tend to grow as we believe to be the case with electrons both behaving the same way in a turbulent environment. In both two of the phenomena we had found a vorticity field $[2,5,6]$.

In order to further prove our point we note that in condition of homogeneity where there are no gradients the bulk modulus becomes:

$$
B=m c^{2} \frac{|\psi|^{2}}{\chi V}=\frac{\alpha|\psi|^{2}}{V}\left(T-T_{c}\right)
$$

The superconducting energies according to Slichter [7] are related to the relativistic energy $\mathrm{E}=\mathrm{mc}^{2}$.

In our case we have a diamagnetic susceptibility instead of dielectric susceptibility, since the potential energy is purely of magnetic nature, which depends like the Curie law:

$$
\chi \approx \frac{C}{\left(T-T_{C}\right)}(12)
$$

The result in relativistic arguments of applying equation (8) is the same since the chi factor appears in the same way in space time metric:

$$
d s^{2}=\frac{c^{2}}{\chi} d t^{2}-d \vec{r}^{2}
$$

The final step is to assign the probability or charge density the thermal charge density:

$$
E P=\frac{d Q}{d V}=\frac{T d S}{d V}=\hbar \omega \frac{d q}{e d V} \Rightarrow d Q=\frac{d q}{e} E
$$

By making use of equation (14) we are able to explain the first term in the right member of the free energy expansion of (9)

$$
\frac{\left(T-T_{c}\right)}{T_{c}} \alpha|\psi|^{2}=\eta \frac{d Q}{d V}=\frac{d W}{d V}=P d V
$$

In equation (15) Greek eta $\mathrm{h}$ is the coefficient of useful work known from Carnot engines. Through combining equations (6), (9), (14), (15) we arrive at the following conclusion:

$$
\frac{d \Omega}{d V}=\frac{1}{2 N} \vec{p}^{*} \cdot \vec{v}
$$

In the more general case we may use formula (16) to give a definition for pressure in quantum mechanics and rewrite Schrodinger's equation as follows:

$$
P=\frac{|\psi|^{2}}{N}(E-U) \Rightarrow-\frac{\hbar^{2}}{2 m N} \psi * \Delta \psi=P
$$

Using equation (17) we may obtain thermodynamic results from volume integration:

$$
\langle U\rangle=\int T d S-\int P d V
$$

\section{Conclusion}

The idea that atoms may have distinct thermodynamic properties is owed first to Einstein and other scientists have continued along this path [8]. The reader may also refer to some papers of Mallick S, Millette PA, Smerlak M, Schrodinger E [9-12] discussing the effect of assigning a constant spacetime curvature to the volume dilation and expansion.

By following the logical steps towards a derivation of Ginzburg Landau equations in superconductivity one is led to the conclusion that there are hidden variables in quantum mechanics. One more outcome is the fact that the formulation of the symbols is chosen as to shed light to the physical quantities. One example which is discussed here is that the velocity of light might be less than the prescribed value in vacuum in an atom [2]. This is why Dirac chose the 
Physical Science \& Biophysics Journal

word bracket and separated into bra and ket leaving the letter $\mathrm{c}$ in between. Later he would talk about the $\mathrm{c}$ numbers and the q numbers.

\section{References}

1. Koutandos S (2020) About a possible derivation of the London equations. Physics Essays 33(2): 208-210.

2. Koutandos S (2020) A discussion about the hidden variables in quantum mechanics. Physics Essays 33(4): 513-514.

3. Beig R, Simon $W$ (1992) On the uniqueness of static perfect-fluid solutions in general relativity. Communications in Mathematical physics 144: 373-390.

4. Tinkham M (2004) Introduction to superconductivity. $2^{\text {nd }}(E d n$.$) , Dover Publications, Mineola Newyork, USA.$

5. Koutandos S (2017) Some reflections on the evaporation and condensation phenomena. IOSR Journal of Applied Physics 9(5): 47-48.
6. Tserkovnayak Y, Zou J (2019) Quantum hydrodynamics of vorticity. Physical Review Research 1(3): 033071.

7. Charles PS (1964) Principles of magnetic resonance. $3^{\text {rd }}$ (Edn.), Harper and Row publishing, New York, USA, pp: 658.

8. Zimmermann HW (2002) Particle Entropies and Entropy Quanta. III. The van der Waals Gas. Z Phys Chem 216(5): 615-639.

9. Mallick S, Jin Suh Y, Chand De U (2016) A spacetime with pseudo-projective curvature tensor. Journal of Mathematical Physics 57(6).

10. Millette PA (2014) Wave-Particle Duality in the Elastodynamics of the Spacetime Continuum (STCED). Progress in Physics 10(4): 255-258.

11. Smerlak M (2012) Diffusion in curved spacetimes. New J Phys 14: 023019.

12. Schrodinger E (1930) On force-free motion in relativistic quantum mechanics. Sitzber preuss Akad Wiss Phys Math Klasse 24; 418-428; Ges Abh 3: 357-368. 